\title{
APPROXIMATION TO A GIVEN FUNCTION BY MEANS OF POLYNOMIALS IN ANOTHER GIVEN FUNCTION*
}

\author{
W. C. RISSELMAN
}

1. Introduction. The problem considered in this note is that of approximating to a given function $f(x)$ in a given finite interval by means of polynomials in another given function $\phi(x)$ so as to minimize the integral of a positive power of the absolute value of the error. The purpose is to extend the results of Jackson in the theory of approximation by means of polynomials to the case in which $x$ is replaced by $\phi(x)$. Questions of convergence are confined to uniform convergence. While some of the facts in connection with the problem can be recognized almost immediately, further study leads presently to material complications. This note will give some of the results which are obtained most readily, and others which by their limitations indicate the directions in which difficulty is encountered. It contains the first steps toward the solution of a general problem whose interest is believed to be so great as to warrant an approach under somewhat strong restrictions. While some of the hypotheses seem artificial they are made because of the necessity of recognizing definite limits beyond which the problem does not appear to lend itself to treatment by the methods employed. The study of the problem of convergence is divided into two sections. First is considered the case in which $\phi(x)$ is monotone; then, the case in which $\phi(x)$ is not monotone.

2. Existence and uniqueness. The following theorem on existence is a corollary of a theorem proved by Jackson. $\dagger$

THEOREM 1. Let $\phi(x)$ be a real function of the real variable $x$ which has the following properties:

A. $\phi(x)$ is bounded and measurable for $a \leqq x \leqq b$.

B. If the set where $\phi$ assumes any $n$ values is excluded, the measure of the complementary set is positive.

Then if $f(x)$ is bounded and measurable on $(a, b)$, and if $m$ is a positive constant, there exists at least one set of coefficients $c$ in

$$
P_{n}[\phi(x)]=c_{0 n}+c_{1 n} \phi(x)+c_{2 n} \phi^{2}(x)+\cdots+c_{n n} \phi^{n}(x)
$$

\footnotetext{
* Presented to the Society, April 7, 1928.

$\dagger A$ generalized problem in weighted approximation, Transactions of this Society, vol. 26 (1924), p. 137.
} 
for which the value of the integral

$$
\int_{a}^{b}\left|f(x)-P_{n}[\phi(x)]\right|^{m} d x
$$

is a minimum. If $m>1$, the minimizing polynomial is uniquely determined.

A polynomial which has the minimizing property will be called an approximating polynomial.

3. $\phi(x)$ monotone. A theorem on convergence of a sequence of approximating polynomials is the following:

THEOREM 2. If $f(x)$ is defined and has a continuous pth derivative on the interval $(a, b)$, and if $\phi(x)$ is defined and has $p$ continuous derivatives on $(a, b)$, the first of which is non-vanishing, then the sequence of approximating polynomials, or any sequence of approximating polynomials if the determination is not unique, converges uniformly to $f(x)$ on $(a, b)$ as $n \rightarrow \infty$ if $m$ satisfies the condition $m \geqq 2 / p$.

If $\phi^{\prime}(x)>0$, if $\phi(a)=A$ and $\phi(b)=B$, and if the hypotheses of the theorem are satisfied, the integral (1) becomes

$$
\int_{A}^{B} g^{\prime}(y)\left|h(y)-P_{n}(y)\right|^{m} d y,
$$

where $g(y)$ is the function defined by the equation $y=\phi(x)$, and $h(y)=f[g(y)]$. The function $g^{\prime}(y)$ is continuous and greater than zero on $(A, B)$ and $h(y)$ has a continuous $p$ th derivative. The proof then makes use of a corollary to Bernstein's theorem on the derivative of a real polynomial, or Markoff's theorem on the derivative of a polynomial, together with a theorem on polynomial representation.*

If $\phi(x)$ is monotone and $\phi^{\prime}(x)$ has zeros, the following theorem holds :

THEOREM 3. If $f(x)$ is defined and has a continuous first derivative on $(a, b)$, if $\phi(x)$ is a monotone function which is defined and has a continuous first derivative which has a finite number of zeros on this interval, and if $f^{\prime}(x) / \phi^{\prime}(x)$ has a finite limit as $x$ approaches a zero of $\phi^{\prime}(x)$, the sequence of approximating polynomials $\left\{P_{n}[\phi(x)]\right\}$ converges uniformly to $f(x)$ on $(a, b)$ as $n \rightarrow \infty$, provided $m \geqq 2$.

* For details the reader is referred to pp. 96 and 97 and the corollary on p. 18 of The Theory of Approximation, by D. Jackson, American Mathematical Society Colloquium Publications, vol. 11, New York, 1930. Hereafter this book will be called J. 
Under these hypotheses the weight function $g^{\prime}(y)$ in (2) has discontinuities. However, the validity of the transformation of (1) into the form of (2) can be established by examining the formulas with consideration of the fact that $g(y)$ is continuous and is the integral of its own derivative. Then one proves that $h^{\prime}(y)$ is a continuous function. Let $(D, E)$ be an interval in which $g^{\prime}(y)$ is continuous at every point except $D$. Let $\lim _{x \rightarrow g(D)} f^{\prime}(x) / \phi^{\prime}(x)=L$. Let $\psi(y)=h^{\prime}(y)$ on $D<y \leqq E, \psi(D)=L$, and

$$
H(y)=h(E)+\int_{E}^{y} \psi(y) d y .
$$

Then $H^{\prime}(y)$ is continuous on the closed interval $(D, E)$, and $H(y)$ $=h(y)$ on this closed interval. It can now be seen that the hypotheses of one of Jackson's theorems are satisfied.*

It is to be noticed that the above hypothesis that $f^{\prime}(x) / \phi^{\prime}(x)$ has a finite limit as $x$ approaches a zero of $\phi^{\prime}(x)$ is very restrictive. It means, for instance, that $f^{\prime}$ vanishes wherever $\phi^{\prime}$ vanishes. But if $f^{\prime} / \phi^{\prime} \rightarrow \infty$ as $x$ approaches a zero of $\phi^{\prime}$, then the first derivative of $h(y)$, the function to be approximated, is discontinuous.

The hypothesis that the derivative of $\phi(x)$ is defined everywhere will now be abandoned. The following theorem holds:

THEOREM 4. If $\phi(x)$ is a monotonic function which is defined for $a \leqq x \leqq b$ and satisfies the condition that there exist positive numbers $k_{1}$ and $k_{2}$ independent of $x$ such that

$$
k_{1}\left|x_{2}-x_{1}\right|^{\beta} \leqq\left|\frac{\phi\left(x_{2}\right)-\phi\left(x_{1}\right)}{x_{2}-x_{1}}\right| \leqq k_{2},
$$

where $a \leqq x_{1}, x_{2} \leqq b, x_{1} \neq x_{2}$, and $\beta \geqq 0$ and independent of $x$; if $f(x)$ is any function which on $(a, b)$, is defined and satisfies a Lipschitz condition of order $\alpha\left|f\left(x_{2}\right)-f\left(x_{1}\right)\right| \leqq \lambda\left|x_{2}-x_{1}\right|^{\alpha}$, where $\lambda$ is independent of $x$, then the sequence of approximating polynomials $\left\{P_{n}[\phi(x)]\right\}$ converges uniformly to $f(x)$ on $(a, b)$ as $n \rightarrow \infty$ provided $\alpha, \beta$, and $m$ satisfy $\alpha /(1+\beta)>2 / m$, and it converges uniformly to $f(x)$ on any closed interval interior to $(a, b)$ as $n \rightarrow \infty$ provided that these quantities satisfy the inequality $\alpha /(1+\beta)>1 / m$.

Under these hypotheses the integral (1) equals the Stieltjes integral

$$
\gamma_{n}=\int_{A}^{B}\left|f[g(y)]-P_{n}(y)\right|^{m} d g .
$$

* J, Theorem VII of chap. 3. 
Suppose there exists a sequence of polynomials $\left\{p_{n}(y)\right\}$ such that $\left|f[g(y)]-p_{n}(y)\right| \leqq \epsilon_{n}$ on $(A, B)$. By using the corollary to Bernstein's theorem which was used before and an iteration process* it can be shown that $\left|f[g(y)]-P_{n}(y)\right|$ does not exceed

$$
5 \epsilon_{n}+d_{1} n^{2 / m} \gamma_{n}{ }^{1 / m}
$$

on $(a, b)$ and

$$
5 \epsilon_{n}+d_{2}\left(n^{v} \gamma_{n}\right)^{1 / m}
$$

on any closed interval interior to $(a, b)$, where $\nu=1+(m+1)^{-k}, k$ is an arbitrary positive integer, and the $d$ 's are constants. In the following manner it is seen that $f[g(y)]$ satisfies a Lipschitz condition of order $\alpha /(1+\beta)$. Since $f(x)$ satisfies the Lipschitz condition of order $\alpha$, if $y_{1}$ and $y_{2}$ are any two values of $y$ on $(A, B)$,

$$
\left|f\left[g\left(y_{2}\right)\right]-f\left[g\left(y_{1}\right)\right]\right| \leqq \lambda\left|g\left(y_{2}\right)-g\left(y_{1}\right)\right|^{\alpha} .
$$

From one of the conditions imposed on $\phi$,

$$
\left|g\left(y_{2}\right)-g\left(y_{1}\right)\right|^{\alpha} \leqq\left(1 / k_{1}\right)^{\alpha /(1+\beta)}\left|y_{2}-y_{1}\right|^{\alpha /(1+\beta)} .
$$

Therefore $f[g(y)]$ satisfies the Lipschitz condition

$$
\left|f\left[g\left(y_{2}\right)\right]-f\left[g\left(y_{1}\right)\right]\right| \leqq \lambda_{1}\left|y_{2}-y_{1}\right|^{\alpha /(1+\beta)},
$$

where $\lambda_{1}=\lambda\left(1 / k_{1}\right)^{\alpha /(1+\beta)}$. Since $f[g(y)]$ satisfies this Lipschitz condition, $\epsilon_{n}$ may be taken equal to $K / n^{\alpha /(1+\beta)}$, where $K$ is a constant, and $\gamma_{n}$ is found to be less than a constant times $n^{-m \alpha /(1+\beta)}$. These expressions are then substituted in (3) and (4).

If $\alpha=1$ and $\beta=0$, the last theorem reduces to the theorem :

If $\phi(x)$ and $f(x)$ are defined for $a \leqq x \leqq b$, if $\phi(x)$ is monotone, and if there exist positive constants $k_{1}, k_{2}$, and $\lambda$ independent of $x$ such that

$$
k_{1} \leqq\left|\frac{\phi\left(x_{2}\right)-\phi\left(x_{1}\right)}{x_{2}-x_{1}}\right| \leqq k_{2}, \quad\left|\frac{f\left(x_{2}\right)-f\left(x_{1}\right)}{x_{2}-x_{1}}\right| \leqq \lambda,
$$

where $x_{1}$ and $x_{2}$ are any two distinct values of $x$ on $(a, b)$, then the sequence of approximating polynomials converges uniformly to $f(x)$ on $(a, b)$ as $n \rightarrow \infty$ if $m>2$, and it converges uniformly to $f(x)$ on any closed interval interior to $(a, b)$ as $n \rightarrow \infty$ if $m>1$.

Under suitable hypotheses, $\uparrow$ including the one that $\phi(x)$ has a con-

* J, pp. 96 and 97 , and J. M. Earl, Polynomials of best approximation on an infinite interval, Transactions of this Society, vol. 32 (1930), p. 3.

$\dagger$ The reader will find that a theorem can be formulated so that its proof depends on Theorem VII in chap. 3 of $\mathrm{J}$. 
tinuous non-vanishing first derivative where $\phi$ is continuous, it is also found that the sequence of approximating polynomials in a monotone $\phi(x)$ converges uniformly to $f(x)$ on $(a, b)$ as $n \rightarrow \infty$ if $m \geqq 2$ in case $\phi(x)$ has a finite number of finite jumps. In this case the integral is transformed to the form (2). If $\phi(x)$ jumps from $C$ to $D$ at a certain value of $x, h(y)$ is taken to be a suitable polynomial of the third degree in $y$ on $(C, D)$, and the hypotheses on $f$ and $\phi$ may be made so that $h(y)$ has a continuous first derivative on $(A, B)$. The weight function is taken to be zero on the open interval $(C, D)$.

4. $\phi(x)$ not monotone.* If $\phi(x)$ has the same value for different values of $x$, so also will any polynomial in $\phi(x)$ for these same values of $x$. Now, if the values of $f(x)$ for these values of $x$ are different, no sequence $\left\{P_{n}[\phi(x)]\right\}$ of approximating polynomials can converge to $f(x)$ for these values of $x$. However, under certain hypotheses it can be proved that such a sequence converges to a suitably defined mean of the values of $f(x)$ corresponding to a single value of $\phi(x)$.

Let $f(x)$ be a function which is defined and continuous and which has a continuous first derivative on $a \leqq x \leqq b$, and let $\phi(x)$ be a function which satisfies the following conditions:

A. It is continuous on $a \leqq x \leqq b$.

B. It has a finite number, say $(q+1)$, of maxima and minima on $(a, b)$.

C. The maxima of $\phi$ are all equal and the minima are all equal.

D. Let the values of $x$, arranged in order of increasing values, for which $\phi$ has maxima or minima be denoted by $a=a_{0}, a_{1}, a_{2}, \ldots$, $a_{q}=b$. Then $\phi(x)$ has continuous first and second derivatives on all intervals $a_{i} \leqq x \leqq a_{i+1},(i=0,1, \cdots, q-1)$.

E. The relation $\left|\phi^{\prime}(x)\right| \geqq k>0$, where $k$ is independent of $x$, holds for all values of $x$ for which $\left|\phi^{\prime}(x)\right|$ is defined.

The integral (1) may be written in the form

$$
\gamma_{n}=\sum_{i=1}^{q} \int_{A}^{B}\left|g_{i}^{\prime}(y)\right|\left|f\left[g_{i}(y)\right]-P_{n}(y)\right|^{m} d y,
$$

where $g_{i}(y)$ is the function inverse to $\phi(x)$ in the $i$ th subinterval and $A$ is the minimum value of $\phi$ and $B$ its maximum value.

Consider the function

$$
H(z, y)=\sum_{i=1}^{q}\left|g_{i}^{\prime}(y)\right|\left|z-f\left[g_{i}(y)\right]\right|^{m} .
$$

* A paper on a different subject, the methods of which nevertheless have a bearing on the rest of this note, is that by $\mathrm{D}$. Jackson entitled On the trigonometric representation of an ill-defined function, Annals of Mathematics, (2), vol. 26 (1924), pp. 8-20. 
If any value on $(A, B)$ is assigned to $y$ there is exactly one value of $z$ which minimizes $H(z, y)$ if $m>1$. If there were two such values, say $z_{1}$ and $z_{2}$, then since the curve $Y=|X|^{m}$ is concave upward,

$$
\begin{array}{r}
\left|\frac{z_{1}+z_{2}}{2}-f\left(g_{i}\right)\right|^{m}<\frac{1}{2}\left\{\left|z_{1}-f\left(g_{i}\right)\right|^{m}+\left|z_{2}-f\left(g_{i}\right)\right|^{m}\right\}, \\
i=1,2, \cdots, q,
\end{array}
$$

and a contradiction would be obtained. Thus $z$ is defined as a singlevalued function of $y$, which will be denoted by $F(y)$. By taking the partial derivative of $H$ with respect to $z$ it is found that the equation

$$
\sum_{i=1}^{q}\left|g_{i}^{\prime}(y)\right|\left|z-f\left[g_{i}(y)\right]\right|^{m-1} \operatorname{sgn}\left\{z-f\left[g_{i}(y)\right]\right\}=0,
$$

defines $z$ as this function of $y$. Under suitable hypotheses it will be proved that the sequence $\left\{P_{n}(y)\right\}$ converges uniformly to $F(y)$ as $n \rightarrow \infty$.

Consider, first, two special cases: (i) $m=2, q$ is arbitrary; (ii) $m>2$, $q=2$, where the explicit expression for $F(y)$ will be given.

(i) $m=2, q$ is arbitrary. If $m=2$, it is found from (5) that

$$
F(y)=\frac{\sum_{i=1}^{q}\left|g_{i}^{\prime}(y)\right| f\left[g_{i}(y)\right]}{\sum_{i=1}^{q}\left|g_{i}^{\prime}(y)\right|} .
$$

The integral $\gamma_{n}$ may be expressed in the form

$$
\gamma_{n}=\int_{A}^{B} \sum_{i=1}^{q}\left|g_{i}^{\prime}(y)\right|\left\{\left(f\left[g_{i}(y)\right]-F(y)\right)+\left(F(y)-P_{n}(y)\right)\right\}^{2} d y .
$$

By expanding this expression and taking (5) into account it is found that

$$
\gamma_{n}=\int_{A}^{B} \sum_{i=1}^{q}\left|g_{i}^{\prime}\right|\left[f\left(g_{i}\right)-F\right]^{2} d y+\int_{A}^{B}\left\{\sum_{i=1}^{q}\left|g_{i}^{\prime}\right|\right\}\left\{F-P_{n}\right\}^{2} d y .
$$

Thus the original problem is reduced to that of approximating to $F(y)$ by means of polynomials in $y$. From the hypotheses it follows that $F(y)$ has a continuous first derivative on $(A, B)$ and that the weight function $\sum_{i=1}^{q}\left|g_{i}^{\prime}\right|$ has a positive minimum on this interval. Thus one has the theorem:*

\footnotetext{
* See J, Theorem VII of chap. 3.
} 
THEOREM 5. If $f(x)$ has a continuous derivative on $(a, b)$, if $\phi(x)$ satisfies the hypotheses $\mathrm{A}$ to $\mathrm{E}$, and if $m=2$, the sequence $\left\{P_{n}[\phi(x)]\right\}$ converges uniformly as $n \rightarrow \infty$ to the function

$$
F[\phi(x)]=\frac{\sum_{i=1}^{q}\left|g_{i}^{\prime}(\phi)\right| f\left[g_{i}(\phi)\right]}{\sum_{i=1}^{q}\left|g_{i}^{\prime}(\phi)\right|} \text { on }(a, b),
$$

where $F[\phi(x)]$ is obtained as follows: Corresponding to any value of $x$ on $(a, b) a$ value of $y$ is determined by means of $y=\phi(x)$. Using this value of $y$ one obtains from $x=g_{i}(y),(i=1,2, \cdots, q), q$ values of $x$, say, $x^{(1)}, x^{(2)}, \cdots, x^{(q)}$. Then $F[\phi(x)]$ is the indicated mean of the values $f\left(x^{(1)}\right), f\left(x^{(2)}\right), \cdots, f\left(x^{(q)}\right)$.

(ii) $m>2, q=2$. If the inverse of $\phi$ is double-valued and $m>2$, the function of $y$ defined by (5) is given by

$$
F(y)=\frac{\left|g_{1}^{\prime}\right| 1 /(m-1) f\left(g_{1}\right)+\left|g_{2}^{\prime}\right|^{1 /(m-1)} f\left(g_{2}\right)}{\left|g_{1}^{\prime}\right|^{1 /(m-1)}+\left|g_{2}^{\prime}\right|^{1 /(m-1)}} .
$$

Again $F(y)$ has a continuous first derivative on $(A, B)$. Let the function $G_{2}(z, y)$ be defined by the equation

$$
G_{2}(z, y)=\sum_{i=1}^{2}\left|g_{i}^{\prime}(y)\right|\left|z-\left\{f\left[g_{i}(y)\right]-p_{n}(y)\right\}\right|^{m},
$$

where $p_{n}(y)$ is the Tchebychef polynomial, of degree $\leqq n$, of best approximation to $F(y)$ on $(A, B)$. Let $u_{i}(y)=f\left[g_{i}(y)\right]-p_{n}(y)$ and let $\Pi_{n}(y)=P_{n}(y)-p_{n}(y)$. Then $u_{i}(y)-\Pi_{n}(y)=f\left[g_{i}(y)\right]-P_{n}(y)$ and $\gamma_{n}=\int_{A}^{B} G_{2}\left[\Pi_{n}(y), y\right] d y$. The second derivative of $G_{2}$ with respect to $z$ is continuous in the region $R:(-M \leqq z \leqq M ; A \leqq y \leqq B)$, where $M$ denotes the maximum of $|F(y)|$ on $(A, B)$. Let $z_{0}=F(y)-p_{n}(y)$ be the value of $z$ for which $G_{2}(z, y)$ is a minimum. Expansion of $G_{2}$ according to Taylor's theorem gives

$$
G_{2}(0, y)=G_{2}\left(z_{0}, y\right)+\frac{1}{2} z_{0}^{2} G_{2}^{\prime \prime}\left(\zeta_{0}, y\right),
$$

where $\zeta_{0}$ is between 0 and $z_{0}$, and $G_{2}^{\prime \prime}$ denotes the second derivative with respect to $z$. Let $\epsilon_{n}$ be the maximum of $\left|z_{0}\right|$ on $(A, B)$. Then $\epsilon_{n} \leqq M$. It follows that $\zeta_{0}$ satisfies $-M \leqq \zeta_{0} \leqq M$ for all $y$ on $(A, B)$. Let the maximum of $G_{2}^{\prime \prime}$ in $R$ be denoted by $2 V$. Then

$$
G_{2}(0, y) \leqq G_{2}\left(z_{0}, y\right)+z_{0}^{2} V
$$

for all $y$ on $(A, B)$. Therefore 


$$
\begin{aligned}
\gamma_{n}=\int_{A}^{B} G_{2}\left(\Pi_{n}, y\right) d y & \leqq \int_{A}^{B} G_{2}(0, y) d y \\
& \leqq \int_{A}^{B} G_{2}\left(z_{0}, y\right) d y+V \epsilon_{n}{ }^{2}(B-A) .
\end{aligned}
$$

Let $\mu_{n}=\left|\Pi_{n}\left(y_{0}\right)\right|$ be the maximum of $\left|\Pi_{n}(y)\right|$ on $(A, B)$. By using Markoff's theorem and the mean value theorem it is found in the usual manner that, if $\mu \geqq 4 \epsilon_{n}$, then $\left|\Pi_{n}-z_{0}\right| \geqq \mu_{n} / 4$ throughout an interval $L$ of length $(B-A) / 4 n^{2}$. Let $K$ be the maximum and $k$ the minimum of $\left|g_{1}^{\prime}(y)\right|+\left|g_{2}^{\prime}(y)\right|$ on $(A, B)$. Let $d>0$ be a fixed number which satisfies $d<k^{1 / m} K^{-1 / m} / 4\left(1+k^{1 / m} K^{-1 / m}\right)$. The part of the interval $L$ on which

$$
\left|f\left[g_{1}(y)\right]-f\left[g_{2}(y)\right]\right| \leqq d \mu_{n}
$$

is considered separately from the rest on which this expression is greater than $d \mu_{n}$. In the first case, use is made of the explicit expressions for $G_{2}\left(\Pi_{n}, y\right)$ and $G_{2}(0, y)$, and in the second case use is made of the expansion

$$
G_{2}\left(\Pi_{n}, y\right)=G_{2}\left(z_{0}, y\right)+\frac{1}{2}\left(\Pi_{n}-z_{0}\right)^{2} G_{2}^{\prime \prime}(\zeta, y),
$$

in order to show that $G_{2}\left(\Pi_{n}, y\right) \geqq G_{2}\left(z_{0}, y\right)+c \mu_{n}{ }^{m}$ throughout $L$, where $c$ is constant and positive. Then it is seen that

$$
\begin{aligned}
\gamma_{n} & \geqq \int_{L}\left[G_{2}\left(z_{0}, y\right)+c \mu_{n}^{m}\right] d y+\int_{c L} G_{2}\left(z_{0}, y\right) d y \\
& =\int_{A}^{B} G_{2}\left(z_{0}, y\right) d y+c \mu_{n}^{m} \frac{B-A}{4 n^{2}},
\end{aligned}
$$

where $\int_{L}$ means the integral over the interval $L$, and $\int_{c L}$ means the integral over the rest of $(A, B)$. From (7) and (8) it follows that

$$
\mu_{n} \leqq\left(\frac{4 V}{c}\right)^{1 / m}\left(n \epsilon_{n}\right)^{2 / m} \quad \text { if } \quad \mu_{n} \geqq 4 \epsilon_{n} .
$$

Now $\left|F(y)-P_{n}(y)\right| \leqq \epsilon_{n}+\mu_{n}$ on $\left(\begin{array}{ll}A & B\end{array}\right)$. Since $F(y)$ has a continuous derivative, $\lim _{n \rightarrow \infty} n \epsilon_{n}=0$. Therefore, $\left|F(y)-P_{n}(y)\right|$ approaches zero uniformly, whether $\mu_{n} \geqq 4 \epsilon_{n}$ or not. One now has the theorem:

THEOREM 6. If $f(x)$ is defined and has a continuous first derivative on $(a, b)$ and if $\phi(x)$ satisfies the hypotheses A to $\mathrm{E}$ with $q=2$, the sequence $\left\{P_{n}[\phi(x)]\right\}$ converges uniformly to $F[\phi(x)]$ on $(a, b)$ as $n \rightarrow \infty$ if $n>2$, where $F$ is given by (6). 
Regarding the general case in which $m>2$ and $q \geqq 3$ one can state the following theorem :

THEOREM 7. If $f(x)$ is a univariant function which is defined on $(a, b)$ and has a continuous first derivative on this interval, and if $\phi(x)$ satisfies hypotheses A to $\mathrm{E}$ with $q \geqq 3$, the sequence $\left\{P_{n}[\phi(x)]\right\}$ converges uniformly to $F[\phi(x)]$ on $(a, b)$ as $n \rightarrow \infty$ if $m>2$, where $F$ is the function defined implicitly by (5).

In this case no explicit expression is obtained for $F(y)$. The function

$$
G(z, y)=\sum_{i=1}^{q}\left|g_{i}^{\prime}(y)\right|\left|z-\left\{f\left[g_{i}(y)\right]-p_{n}(y)\right\}\right|^{m},
$$

where $p_{n}(y)$ is the Tchebychef polynomial corresponding to $F(y)$, is introduced. The hypothesis of univariance of $f(x)$ is used to prove that $F^{\prime}(y)$ is continuous on $(A, B)$ and to show that $G^{\prime \prime}(z, y)$ has a positive lower bound in the region $R^{\prime}:(A \leqq y \leqq B ;-\infty<z<\infty)$. The expansion

$$
G(z, y)=G\left(z_{0}, y\right)+\frac{1}{2}\left(z-z_{0}\right)^{2} G^{\prime \prime}(\zeta, y)
$$

is used to compare $G(0, y)$ and $G\left(\Pi_{n}, y\right)$ with $G\left(z_{0}, y\right)$. The rest of the proof is similar to the corresponding parts of the proof of the theorem immediately preceding this one.

In case $m=1$, it can be shown, by methods that offer no essential novelty, that the sequence of approximating polynomials converges uniformly to $F[\phi(x)]$ in any finite set of closed intervals not containing points where $\phi$ has maxima or minima, provided $f$ and $\phi$ satisfy suitable hypotheses under which $\phi$ has a continuous first derivative everywhere on $(a, b)$, this derivative not being required to be everywhere different from zero. However, these hypotheses exclude functions $\phi$ with a continuous second derivative. In special cases it is found that convergence occurs even in this case. For example, if the interval is $(-1,1)$, then $P_{n}\left(x^{2}\right)$ is the partial sum of the Legendre series for $E(x)=\frac{1}{2}[f(x)+f(-x)]$ taken through the term involving $\phi_{2 n}(x)$, where $\phi_{i}(x)$ is the $i$ th normalized Legendre polynomial.

University of California at Los Angeles 